WITOLD UCHEREK

Université de Wrocław

\title{
LES LISTES DE NOMS PROPRES DANS LES DICTIONNAIRES GÉNÉRAUX POLONAIS-FRANÇAIS ET FRANÇAIS-POLONAIS
}

\section{INTRODUCTION}

Comme le remarque à juste titre Carla Marello, «par rapport à la macrostructure des dictionnaires monolingues généraux, les bilingues généraux devraient contenir aussi un certain nombre de noms propres de personnes et de lieux, surtout quand ces noms diffèrent d'une langue à l'autre ${ }^{1}$. Or, un lexicographe bilingue désireux d'inclure des noms propres dans son dictionnaire a plusieurs possibilités. En effet, il peut non seulement les insérer dans la nomenclature, mais aussi les grouper dans une liste ou une table à part, ou bien en placer une partie dans la nomenclature et une autre dans une annexe.

Notre objet de recherche est d'examiner les listes de noms propres figurant dans les dictionnaires bilingues généraux polonais-français et français-polonais. Plus précisément, nous voudrions mettre en évidence la présence de ces listes dans les bilingues consultés, évaluer le rapport entre elles et les nomenclatures des dictionnaires respectifs, identifier des classes de noms propres listés et voir dans quelle mesure les informations contenues dans les inventaires analysés sont susceptibles de satisfaire les attentes des usagers des dictionnaires dont ils proviennent.

${ }^{1}$ C. Marello, « Les différents types de dictionnaires bilingues », [dans :] H. Béjoint et Ph. Thoiron (dir.), Les dictionnaires bilingues, Aupelf-Uref — Éditions Duculot, Louvain-la-Neuve 1996, p. 37. 
Les observations qui suivent découlent de la consultation de 45 dictionnaires de toutes les tailles, publiés au cours des 150 dernières années. Leurs références, accompagnées des abréviations identifiant ces ouvrages, se trouvent à la fin de l'article.

\section{LA PLACE DES LISTES LEXICALES DANS L'ARCHITECTURE DU DICTIONNAIRE}

À la suite de Josette Rey-Debove, dans la lexicographie française, il est de coutume de distinguer deux différentes structures dans un dictionnaire : la microstructure, c'est-à-dire " l'ensemble des informations ordonnées de chaque article » et la macrostructure. Cette dernière est définie par Rey-Debove comme «l'ensemble des entrées ordonnées »; l'auteur remarque ensuite que « la macrostructure est couramment nommée nomenclature $»^{2}$, ce qui reste vrai jusqu'à nos jours. En effet, c'est ainsi que le terme est employé par exemple par Michèle Fourment-Berni Canani, qui l'entend comme suit : " La macrostructure, à savoir l'ensemble des entrées figurant dans un dictionnaire $»^{3}$. Cependant, d'autres chercheurs contemporains mettent l'accent sur l'idée d'organisation des mots-vedettes, présente dans la définition précitée, et distinguent soigneusement entre la nomenclature et la macrostructure d'un dictionnaire, comprise alors uniquement comme « la manière dont est ordonnée sa nomenclature (par exemple, la macrostructure alphabétique, la macrostructure à nids) $»^{4}$.

En outre, tout dictionnaire comporte un certain nombre d'éléments paratextuels, hors nomenclature, tels les avis aux lecteurs, préfaces, tableaux de conjugaison, listes d'abréviations, sans oublier les listes lexicales. Certains spécialistes considèrent tous ces éléments comme faisant également partie de la macrostructure. Citons ici Vladimir Grigorievitch Gak, qui comprend le terme dans son acception large : « la macrostructure embrasse toute l'architecture du dictionnaire, y compris la préface, les tableaux et les illustrations s'il y en a, les annexes, etc. $»^{5}$. Dans ce troisième sens, le terme de macrostructure est actuellement concurrencé

2 J. Rey-Debove, Étude linguistique et sémiotique des dictionnaires français contemporains, Mouton, The Hague \& Paris 1971, p. 21.

${ }^{3}$ M. Fourment-Berni Canani, « Le dictionnaire bilingue : de la médiation linguistique à la médiation didactique », Le Français dans le Monde, numéro spécial : La médiation et la didactique des langues et des cultures, janvier 2003, p. 74.

${ }^{4}$ D. Melnikienè, Le dictionnaire bilingue. Un miroir déformant?, Hermann, Paris 2013, p. 171. Voir aussi : J. Pruvost, Les dictionnaires français outils d'une langue et d'une culture, Ophrys, Paris 2006, pp. 160 et 192.

${ }^{5}$ V.G. Gak, « De la langue-système à la réalisation discursive dans un dictionnaire bilingue "de type actif" ", [dans :] H. Tommola et al. (dir.), Euralex '92 Proceedings II, University of Tampere 1992, p. 329. Cf. A. Frączek et R. Lipczuk, Stowniki polsko-niemieckie i niemiecko-polskie. Historia i teraźniejszość, Oficyna In Plus, Wołczkowo 2004, p. 17. 
par celui de mégastructure, d’origine anglaise. Dangoulè Melnikienè explique que «c'est la structure générale du dictionnaire, parfois appelée structure encadrante, qui englobe la macrostructure $[. .$.$] et tous ses éléments paratextuels [. ..] »^{6}$. Ajoutons encore que dans la terminologie lexicographique anglaise, ces différents éléments paratextuels sont appelés en bloc outside matter et qu'ils se laissent subdiviser, en fonction de leur position par rapport au corps principal du dictionnaire, en front matter (par ex. pages de titre, tables des matières, modes d'emploi du dictionnaire), middle (mid-) ou inside matter (par ex. illustrations, encadrés) et back ou end matter (par ex. listes de proverbes, de citations, de poids et mesures) ${ }^{7}$.

Autrement, certains de ces constituants de la mégastructure, situés avant le texte principal, « sont inséparables de tout dictionnaire, car ils l'expliquent ${ }^{8}$ tandis que la présence des autres, renseignant le plus souvent sur la ou les langue(s) traitée(s) dans un dictionnaire donné, reste facultative. Tel est justement le cas des listes lexicales.

En outre, l'information sur la disposition habituelle de différentes listes après le corps principal n'est vraie que pour les dictionnaires monolingues ou les bilingues composés d'une seule partie ; dans ce cas, on peut effectivement parler d'une « méthode de la liste de fin d'ouvrage ${ }^{9}$. En revanche, lorsqu'un bilingue monovolume comporte deux parties (langue $\mathrm{A} \rightarrow$ langue $\mathrm{B}$ et langue $\mathrm{B} \rightarrow$ langue $\mathrm{A}$ ), il arrive parfois que des listes ou autres éléments de mégastructure les séparent. Quant aux dictionnaires du corpus, il en est ainsi par exemple dans les DU, JST, SM et SZT.

\section{LE CONTENU DES LISTES LEXICALES DANS LES DICTIONNAIRES DU CORPUS}

Parmi les dictionnaires dépouillés, il y en a 17 (BI, DEF, DEP, HAF, HAP, KAF, KAP, KS, KU, LAN, LAP, LARF, LAW, LIF, LIP, NM, POM) qui ne contiennent aucune liste en annexe. Remarquons en passant que ces ouvrages datent d'époques différentes (les premières éditions du HAF et du HAP remontent à 1935 alors que le LAN est paru en 2012) et varient considérablement en taille : on y trouve des mini-dictionnaires, tels le BI ou le POM qui comprennent respectivement 10000 et 15000 mots et expressions, mais aussi des ouvrages plus

${ }^{6}$ D. Melnikienè, op. cit., p. 171.

${ }^{7}$ Pour en savoir plus, consulter R.R.K. Hartmann et G. James, Dictionary of Lexicography, Routledge, London \& New York 2001, passim; I. Burkhanov, Lexicography. A dictionary of Basic Terminology, Rzeszów [1998] 2010, passim ; B.T.S. Atkins et M. Rundell, The Oxford Guide to Practical Lexicography, Oxford University Press, Oxford 2008, pp. 176-177 ; H. Yong et J. Peng, Bilingual Lexicography from a Communicative Perspective, John Benjamins Publishing Company, Amsterdam \& Philadelphia 2007, pp. 83-92.

${ }^{8}$ D. Melnikienè, op. cit., p. 171.

9 S. Leroy, Le Nom propre en français, Ophrys, Gap \& Paris 2004, p. 49. 
volumineux, comme le LAP (30 000 mots et expressions) et surtout le LARF, comptant 50000 mots et expressions, bien qu'il ne comprenne qu'une seule partie, français-polonais.

Par contre, 28 bilingues contiennent chacun au moins une liste lexicale. Toutefois, dans six d'entre eux (BER, MC, POU, PWN, SPR, SS), il n'y a aucune liste de noms propres, ces derniers étant inclus dans la nomenclature de ces ouvrages ; les listes qui y sont présentées répertorient avant tout des adjectifs numéraux cardinaux et ordinaux.

En ce qui concerne les classes référentielles des noms propres listés dans les 22 dictionnaires restants, viennent en tête les noms géographiques, attestés dans tous ces ouvrages, suivis des gentilés (dans 9 dictionnaires) et des prénoms (dans 8 dictionnaires). Ceci n'étonne guère dans la mesure où les noms de lieux et de personnes sont considérés comme le noyau de la catégorie des noms propres ${ }^{10}$. À titre plutôt exceptionnel, il existe aussi des listes reprenant les noms des signes du zodiaque ( 2 fois), des vins et eaux-de-vie français ( 2 fois), des personnages historiques, mythologiques, bibliques, religieux et littéraires ( 1 fois), des monuments historiques, édifices et lieux connus (1 fois) et des corps célestes (1 fois).

\section{APERÇU DES LISTES DE NOMS PROPRES}

\subsection{LISTES DES NOMS D’ALCOOLS}

Les listes en question figurent uniquement dans deux dictionnaires de Mirosława Słobodska, le SPO et le DU, adressés à des polonophones. Elles recensent exactement les mêmes 41 noms d'alcools, et ceci, bien que l'inventaire du SPO, le plus ancien des deux ouvrages, soit intitulé tout simplement Vins alors que celui du DU porte le titre Vins, eaux-de-vie; c'est ce dernier titre qui est le plus conforme au contenu.

Dans ces listes, il y a deux types de dénominations. Les plus nombreuses sont celles venant d'un lieu géographique, par exemple la Champagne, Cognac, Gaillac ou le Minervois, qui est utilisé, par métonymie, pour l'appellation d'origine de la boisson alcoolique. À ce propos, Sarah Leroy remarque que l'on peut « rencontrer des noms propres dépourvus de majuscule initiale. Il s'agit principalement de noms de personnes ou de régions associés à un produit (poubelle, watt, bourgogne, brie, damas, jersey) [...], ce qui fait souvent dire que ces noms propres sont devenus des noms communs "; l'auteur s'abstient de trancher elle-même sur le statut précis des noms de ce type ${ }^{11}$. En tout cas, conformément à la règle

10 Ibidem, p. 32.

11 Ibidem, p. 9. 
générale, non respectée dans les SPO et DU, « lorsqu'un nom de lieu désigne un objet en rapport avec ce lieu, il perd sa majuscule $»^{12}$.

Ensuite, plus rares, il y a des appellations non motivées par un nom géographique, comme cidre, kirsch (écrit sans le «c » dans les deux sources), marc, muscadet ou muscat, qui s'écrivent, à plus forte raison, avec une minuscule. Telle est, bien entendu, la pratique lexicographique, observée aussi bien dans des monolingues français que dans des bilingues français-polonais ( $c f$. GDF). Aussi, nous ignorons pourquoi, en dressant ses listes, l'auteur a présenté tous les 41 noms de boissons avec une majuscule initiale.

En outre, certains des noms des listes analysées, tels champagne, cidre ou marc, font partie de la nomenclature des deux ouvrages, dans laquelle on trouve également d'autres noms d'alcools, curieusement absents des listes, comme bordeaux, cassis ou pastis. Cette fois, la minuscule est de règle.

En raison de cette confusion entre les noms communs, tel bordeaux, et les noms propres utilisés pour indiquer la provenance d'un alcool ( $c f$. vins de Bordeaux, de Bourgogne), un lecteur de ces listes pourrait être porté à croire que, par exemple, dans les phrases Veux-tu un petit marc ou Je vous propose un bourgogne, un côtes-du-rhône ou un sauternes, tous les substantifs s'écrivent avec une majuscule (à moins qu'il ne tombe par hasard sur l'article cognac où figure l'exemple boire un cognac, suivi de sa traduction polonaise (SPO, DU)). De plus, faute de transcription phonétique des noms français listés, il sera par exemple enclin à prononcer le [k] final dans marc, par analogie avec le prénom Marc. D'ailleurs, c'est cette prononciation fautive qui est donnée à l'article marc figurant dans le corps des deux dictionnaires.

Il s'ensuit que les deux listes, loin de dissiper des doutes de nature orthographique - ce qui, reconnaissons-le, n'est pas toujours possible étant donné notamment que « l'usage est flottant si les scripteurs continuent de sentir le rapport de l'objet et du lieu $»^{13}$ — font naître plusieurs questions à ce propos, du moins chez un apprenant averti, et ignorent entièrement la prononciation des noms listés ; visiblement, la description encyclopédique y prend le dessus sur celle linguistique. En effet, mise à part l'équivalence cognac — koniak, tous les noms cités sont suivis d'une courte explication en polonais, précisant le type d'alcool (vin, liqueur, eau-de-vie) et sa région d'origine (par ex. Irouleguy — biate i różowe wina z regionu Pirenejów ('vins blanc et rosés de la région des Pyrénées')). Ainsi, ces listes aident les dictionnaires à remplir tant bien que mal leur mission de médiateur culturel (« les célèbres vins français » sont mentionnés dans la préface du SPO et l'existence d'une liste spéciale souligne encore plus leur importance), tout en négligeant la description purement linguistique, qui est tout de même leur vocation première. Par-dessus tout, vu que les inventaires des noms d'alcools présents

12 M. Grevisse, A. Goosse, Le bon usage, Duculot, Paris-Louvain-la-Neuve 1993, p. 108.

${ }^{13}$ Ibidem. 
dans les listes et dans le corps des dictionnaires se recoupent partiellement et que la nature des renseignements fournis aux deux endroits n'est pas identique, les indications d'ordre linguistique ne figurant que dans les articles lexicographiques, il serait peut-être mieux de rassembler toutes les informations utiles concernant un nom donné dans un seul endroit du dictionnaire, à savoir dans l'article.

\subsection{LISTES DES NOMS DES SIGNES DU ZODIAQUE}

Ces listes, tout comme les précédentes, n'apparaissent que dans le SPO et le DU. Dans les deux ouvrages, les signes du zodiaque ne sont pas énumérés dans l'ordre alphabétique, mais dans un ordre traditionnel qui fait commencer le zodiaque par le signe du Bélier. Pour chaque signe, on donne d'abord le nom français, précédé de l'article défini et suivi d'une transcription phonétique, puis son équivalent polonais. Nous voyons d'emblée que, contrairement à l'opinion que dans un bilingue, la présence des listes de noms propres « est due presque uniquement à la correspondance orthographique ${ }^{14}$, ces inventaires peuvent apporter d'autres informations utiles, en l'occurrence d'ordre phonétique et morphologique.

Toutefois, rien n'empêche d'inclure ces noms dans la nomenclature, ce que l'auteur fait d'ailleurs, bien que sporadiquement. Ainsi, dans les nomenclatures du SPO et du DU, on trouve la vedette Capricorne, accompagnée de son correspondant Koziorożec (dans le SPO, les deux mots sont à tort écrits avec des minuscules). Ensuite, le DU cite le Bélier et le Cancer dans les articles consacrés aux noms communs homophones, respectivement bélier et cancer; ceci n'étonne pas vraiment, vu qu'extraire les noms propres des articles portant sur les noms communs correspondants et les placer en annexe est un procédé assez artificiel, obligeant de répéter l'information sur le genre et la prononciation. En plus, le traitement des noms propres dans un article lexicographique permet l'introduction des exemples de leur emploi ; dans le cas étudié, il serait utile de renseigner un usager polonophone qu'en français, on peut dire par exemple Je suis Verseau ou bien Je suis né sous le signe du Verseau, mais non *Je suis de dessous/de sous le signe $d u$ Verseau, ce qui serait un calque de la construction polonaise Jestem spod znaku Wodnika. En outre, c'est dans les articles bliźnięta et strzelec qu'il serait naturel d'avertir le lecteur que lorsque ces mots désignent un signe du zodiaque, ils se traduisent autrement que par jumeaux et tireur, ce qui n'est pas le cas dans les SPO et DU. Bref, nous ne voyons aucune raison qui justifie d'isoler les noms des signes du zodiaque dans une liste à part, à moins que ce ne soit un besoin de singularité.

${ }^{14}$ M. Lo Nostro, «Texte et paratexte à la recherche du mot perdu », [dans :] G. Dotoli, P. Ligas et C. Boccuzzi (dir.), Ordre et désordre du dictionnaire, Hermann Éditeurs, Paris 2012, p. 214. Toujours à propos de l'orthographe, signalons en passant que dans le SPO et le DU, le mot Sagittaire est écrit avec un seul « $\mathrm{t} »$. 


\subsection{LISTE DES NOMS DE CORPS CÉLESTES}

Cette liste originale n'est présente que dans le GDP ${ }^{15}$; son titre exact est « Corps célestes, adjectifs appropriés, habitants (hypothétiques), etc. ». Elle se limite à seulement onze unités, à savoir le nom Stońce ('Soleil'), les noms des huit planètes du système solaire ainsi que les noms Księżyc ('Lune') et Pluton; ce dernier nom désigne une planète naine, considérée par le passé comme neuvième planète du système solaire. Malgré sa brièveté, cette liste de phénonymes ${ }^{16}$ inspire quelques remarques.

Tout d'abord, il convient de noter que la moitié des noms qui y figurent, à savoir Księżyc, Pluton, Stońce, Uran, Wenus et Ziemia ('Terre') sont également présents dans la nomenclature du GDP. Ensuite, en tant qu'élément de la liste, chacun des onze noms donne lieu à une sorte de petit article dont la structure maximale est la suivante : nom propre polonais et son genre grammatical, son équivalent français et son genre, adjectif dérivé polonais, adjectif dérivé français (avec la forme du féminin), noms d'habitants polonais et français (au masculin et au féminin). Lorsqu'un adjectif n'existe pas en polonais, soit dans cinq cas, l'auteur cite quand même la forme française, par exemple mercurien ou neptunien. Dans un cas, l'absence de l'adjectif polonais n'est pas justifiée car le mot jowiszowy ('jovien') existe bel et bien, figurant d'ailleurs dans la nomenclature du dictionnaire, tout comme cinq autres adjectifs, tels marsjański ou wenusjański. On en vient à constater que la liste examinée n'est pas très bien coordonnée avec la nomenclature et le contenu des articles du GDP. Enfin, pour ce qui est des noms d'habitants inexistant en polonais, on donne les constructions mieszkaniec/mieszkanka ('habi$\operatorname{tant}(\mathrm{e})$ de') + nom du corps céleste, suivies des noms français (par ex. Neptunien). La solution adoptée pour les adjectifs et les noms d'habitants permet d'informer l'usager du dictionnaire sur un contraste lexical entre les deux langues.

Ainsi, la liste contient plusieurs informations liées aux connaissances linguistiques. Ces dernières pourraient naturellement être complétées par des indications phonétiques. En outre, il serait utile de placer les noms qui la composent dans un contexte. Force est d'évoquer ici l'exemple na Marsie - sur la Mars venant du POP et du POW (s.v. $n a)^{17}$, qui prouve que même les lexicographes peuvent se tromper en utilisant un nom de planète. Seulement, l'ajout de nouveaux éléments

15 Toutes les listes des noms propres du GDP ont été élaborées par Ewa KrajewskaWojciechowska.

${ }^{16}$ Nous reprenons le terme proposé par Gerhard Bauer dans son livre Namenkunde des Deutschen (Peter Lang, Bern 1985) et employé par Leroy, qui explique que les phénonymes sont les noms propres des phénomènes naturels, tels ouragans, tempêtes, cyclones, mais aussi « astres et planètes considérés en tant que tels (Pluton, Neptune)» (S. Leroy, op. cit., p. 34).

${ }^{17}$ Bien que les POP et POW portent des titres différents et annoncent un autre nombre d'articles et expressions contenus (respectivement 60000 et 70 000), ces deux dictionnaires PONS sont identiques quant à leur nomenclature et la microstructure des articles. 
transformerait définitivement cette liste en une succession d'articles à part entière, que l'on pourrait situer sans entrave dans le corps du dictionnaire.

\subsection{LISTE DES NOMS DE MONUMENTS HISTORIQUES, ÉDIFICES ET LIEUX CONNUS}

Une autre innovation du GDP consiste à regrouper dans une liste environ 200 noms de monuments historiques, édifices et lieux connus du monde entier ${ }^{18}$, c'està-dire des noms appartenant à la grande catégorie des toponymes ${ }^{19}$. Quant au choix de ces unités, il est visiblement subjectif dans la mesure où on y trouve par exemple Bazylika Świętego Piotra ('basilique Saint-Pierre'), Wieża Eiffla ('tour Eiffel') ou Ermitaż ('Ermitage'), mais aussi Instytut Smolny ('Institut Smolny'; le GDP propose en guise de correspondant français Smolny college (sic !)), Sing Sing ou bien Czarny Kot ('Chat noir'), sans doute un peu moins célèbres. En outre, par exemple Eskwilin ('Esquilin') fait tout de suite penser à Kwirynat ('Quirinal'), absent de cet inventaire. Pareillement, on y trouvera Kościót Marii Magdaleny ('église de la Madeleine', à Paris), mais non Kościót Mariacki ('église NotreDame', à Cracovie), Trójkąt Bermudzki ('Triangle des Bermudes'), mais non Ztoty Trójkąt ('Triangle d'or'), etc. Par ailleurs, plutôt que de donner des équivalences comme Broadway - Broadway, Bronx - Bronx ou Brooklyn - Brooklyn, heureusement très peu nombreuses, pourquoi ne pas ajouter par exemple Zakazane Miasto ('Cité interdite') ou Usta Prawdy ('Bocca della Verità') ? Aussi, il serait intéressant de savoir quels ont été les principes de sélection des noms pour la liste.

Au plan purement formel, les unités recensées sont soit des mots graphiques simples (par ex. Alhambra), soit des mots constitués de deux ou plusieurs membres. Les premières n'apparaissent pas dans la nomenclature du GDP, tandis que les secondes, à condition qu'elles contiennent au moins un nom commun, sont parfois citées dans l'article qui lui est consacré. Normalement, ces mots polylexicaux ont alors le statut de simples exemples illustrant une vedette ( $c f$. Biały Dom - la Maison-Blanche, s.v. biały et dom) ; plus rarement, ils sont identifiés (par un losange) comme des phraséologismes ( $c f$. kraj < kraina $>$ wschodzacego słońca Empire du Soleil Levant (s.v. wschodzacy). Cette redondance permet de comparer les équivalences de la liste analysée avec celles proposées dans la microstructure des articles du GDP. Or, certaines différences se laissent observer, concernant tant la forme des noms polonais que celle de leurs correspondants français. À titre d'exemple, la construction Grób Nieznanego Żotnierza reçoit dans le GDP les équivalents suivants : Tombeau du Soldat Inconnu (s.v. grób), Le Tombeau $d u$ Soldat inconnu (s.v. nieznany) et tombe du Soldat Inconnu (liste). Ensuite, pour la dénomination du Japon, outre le couple cité plus haut, nous avons relevé :

${ }^{18}$ Une des équivalences listées, Amazonka - Amazone, devrait figurer sur une autre liste, à savoir celle d'anthroponymes.

${ }^{19}$ Cf. S. Leroy, op. cit., p. 34. 
kraina wschodzacego stońca - pays <empire > du Soleil levant (s.v. kraina) et Kraina <Kraj> wschodzacego stońca - Pays du soleil levant (liste) ; empressons-nous de dire que la norme polonaise impose la graphie avec des majuscules, soit Kraina/Kraj Wschodzacego Stońca ${ }^{20}$. De tels exemples pourraient être multipliés. Toujours est-il qu'on observe à plusieurs reprises un flottement dans les variantes des membres de ces appellations, et surtout dans leur orthographe, ce qui peut être partiellement expliqué par l'usage flottant des majuscules dans les noms propres composés, et ce, aussi bien en polonais qu'en français.

Autrement, les noms listés sont régulièrement accompagnés d'abréviations grammaticales précisant le genre des substantifs, et parfois aussi de marques de domaine (antique, mythologie, histoire) et stylistiques (familier, poétique). En plus, l'auteur se sert, s'il le juge utile, d'explications encyclopédiques entre parenthèses, mentionnant le plus souvent où se trouve le monument (par ex. w Londynie ('à Londres')) ou précisant son caractère (par ex. prison, grotte, obélisque); de plus, il explique quelquefois quel est le référent du nom (par ex. o Rzymie ('en parlant de Rome')). Les renseignements de ce type ne figurent jamais dans la microstructure des articles qui citent certains des noms de la liste.

Tout compte fait, inclure dans le dictionnaire bilingue un échantillon des noms dénotant des monuments ou endroits connus du monde entier est une chose très utile. Toutefois, comme c'est le cas pour d'autres classes de noms propres étudiées précédemment, rien n'empêche de situer ces noms dans le corps du dictionnaire, d'autant plus que certains y sont déjà.

\subsection{LISTES DES PRÉNOMS}

Les prénoms font l'objet d'une liste dans huit ouvrages du corpus, dont cinq dictionnaires comportant deux parties en un volume (CA, DU, MB, SPO, SZT), et trois autres composés d'une seule partie (DA, GDP, JA). Toutefois, parmi les dictionnaires les plus anciens, seul le $\mathrm{CA}$ les regroupe dans une liste à part ; dans les DA et JA, ils se retrouvent dans des listes collectives rassemblant les prénoms, qui y sont appelés noms propres, et les noms géographiques. En revanche, dans les dictionnaires contemporains, les prénoms font objet d'une liste à part, à la seule exception du GDP, mais pour une autre raison. C'est que cet ouvrage propose une ample liste de « noms propres, personnages historiques, mythologiques, bibliques, religieux, littéraires et autres ", comprenant aussi des noms tels que Czerwony Kapturek ('Le Petit Chaperon rouge'), Czyngis-chan ('Gengis Khân'), Muminek ('Moomin, Moomintroll'), Merowingowie ('les Mérovingiens'), Zorro, etc. La liste contient au total plus de 1200 unités.

${ }^{20}$ Cf. P. Żmigrodzki (dir.), Wielki słownik języka polskiego, <http://www.wsjp.pl/>. De même, on écrira Kraj/Kraina Kwitnacej Wiśni, qui est une autre dénomination du Japon, absente toutefois de la liste du GDP. 
Au point de vue de leur organisation, ces listes divergent légèrement. En effet, les prénoms commençant par la même lettre y sont parfois isolés par un saut de ligne ( $c f$. GDP, SZT), les lettres successives pouvant alors être citées en guise d'intitulé des sections ( $c f$. DA, JA, MB), ce qui renforce la lisibilité de la liste et facilite le repérage d'un prénom donné. En outre, le MB offre deux listes en séparant les prénoms féminins et masculins, ce qui n'est pas une bonne solution du point de vue didactique.

Quant à leur longueur, les différences sont assez importantes. Pour ce qui est des deux inventaires polonais-français restants, ils comptent respectivement quelque 130 (SZT) et 230 (CA) unités ; cet écart s'explique partiellement par la taille des deux ouvrages et le fait que le CA, en plus des formes officielles, liste également certains hypocoristiques, tels Jaś ou Joasia. En ce qui concerne les inventaires français-polonais, leur importance numérique va d'environ 170 unités dans le SZT ou 190 dans le CA jusqu'à 270 dans les DU et SPO. Certes, le $\mathrm{MB}$ cite presque 450 prénoms français, mais ne les accompagne pas de leurs équivalents polonais, ce qui surprend dans un dictionnaire censé servir de pont entre deux langues. La comparaison du CA et du SZT démontre que le premier dictionnaire inclut plus d'unités dans la liste polonaise que dans la française, à l'inverse du second. En général, on peut se demander quels sont les critères de choix des prénoms, puisque vu leur nombre ${ }^{21}$, même le GDP n'a pas pu les reprendre tous. Aucun ouvrage du corpus ne s'explique d'ailleurs à ce sujet. En tout cas, dans le GDP, ce n'est pas le critère de fréquence qui a été adopté. En effet, sont absents de la liste des prénoms tels que Zbigniew, Mirosław, Stawomir, Wiestaw ou Przemysław, figurant parmi la quarantaine de prénoms masculins les plus populaires en Pologne ${ }^{22}$. On pourrait supposer que l'auteur de la liste les a omis tout simplement parce qu'ils n'ont pas d'équivalent français, alors que toutes les unités listées en ont, ce qui crée une fausse image de correspondance parfaite entre le polonais et le français. Par ailleurs, tous ces prénoms composés, qui datent d'avant la christianisation de la Pologne, font partie de son patrimoine culturel, et dès lors, au-delà de leur fréquence actuelle (qui varie selon les cas), leur insertion dans un bilingue polonais est pleinement justifiée, peu importe que ce soit dans une liste ou dans la nomenclature.

Pour en revenir au problème de correspondance, la solution de Słobodska, qui marque d'un astérisque les prénoms n'ayant pas d'équivalent (par ex. Gaston, Noëlle) et répète la forme française à l'endroit prévu pour son homologue polonais,

${ }^{21}$ L'index polonais des prénoms du Wiktionnaire (https://pl.wiktionary.org/wiki/Indeks:Polski_Imiona) contient environ 1200 unités (sans compter les diminutifs).

22 Cf. T. Walczak, 300 najpopularniejszych imion męskich, Agora SA, Warszawa 2008, pp. 89-90. Remarquons que le dernier volume du GDP, comprenant la liste analysée, a été publié la même année que le livre de Walczak. 
paraît nettement meilleure ( $c f$. DU, SPO $)^{23}$. Ajoutons encore que les DU et SPO sont les seuls à donner systématiquement la prononciation des prénoms français listés, ce qui constitue un autre atout de ces dictionnaires. En revanche, il est dommage qu'il n'y ait pas une seconde liste, allant du polonais vers le français. En son absence, trouver l'équivalent français d'un prénom polonais devient compliqué, d'autant plus que ces équivalents ne commencent pas toujours par la même lettre que la forme de départ (cf. Wojciech - Adalbert, Wilhelm - Guillaume, Iwona - Yvonne). Cette remarque reste pertinente aussi pour certains toponymes ( $c f$. Niemcy - Allemagne, Wegry - Hongrie ou Włochy - Italie) et concerne des dictionnaires tels que le RO ou le SPO (voir point 4.6.).

Ensuite, on aimerait que la présence des formes hypocoristiques soit plus grande dans ces listes, tant pour les prénoms polonais que français. Ce qui est frappant, c'est que le plus grand choix d'hypocoristiques polonais est cité dans le $\mathrm{CA}$, dictionnaire conçu il y a plus d'un siècle par Oscar Callier, philologue et enseignant, sachant visiblement apprécier cette richesse de notre langue. De même, les hypocoristiques français, tels Dédé ou Fifi ( $c f$. DPO et DU), sont trop peu nombreux dans les sources examinées. Or, leur association avec les formes canoniques, en l'occurrence André et Sophie ou Joséphine, peut poser des problèmes aux apprenants polonophones.

Finalement, on observe quelques tentatives de construire un vrai article lexicographique autour d'un prénom listé. Ainsi, le CA, après avoir proposé deux équivalents du prénom Guy (Gwidon et Wit), présente au lecteur l'équivalence la danse de Saint-Guy - choroba świętego Wita ; à Job, on trouve les locutions comparatives pauvre comme Job - goty jak turecki święty. En revanche, les développements relevés dans le GDP ne portent jamais sur l'aspect phraséologique mais sur des personnages connus portant le prénom en question. Par exemple, à Bolestaw, on trouve un petit inventaire de noms de quatre rois de Pologne portant ce prénom, suivis de leurs traductions françaises, tel Bolesław I Chrobry Boleslas $I^{e r}$ dit le Vaillant. Et rien d'étonnant à ce que certains dictionnaires ( $c f$. HAF, HAP), au lieu d'en dresser une liste, incluent les prénoms dans leur nomenclature de noms communs.

\subsection{LISTES DES NOMS GÉOGRAPHIQUES}

Comme nous l'avons déjà signalé au point 3., des listes de noms géographiques sont fournies dans 22 dictionnaires du corpus, soit dans tous ceux qui listent des noms propres ; celle du AS a en réalité la forme d'un tableau offrant « les noms des pays et des régions du monde avec l'adjectif, le nom de nationalité et la (ou les) langue(s) principale(s) correspondant(s), en français et en

${ }^{23}$ Certains prénoms présentés comme sans équivalent polonais en possèdent un en réalité : $c f$. Nicole - Nikola ou Jacqueline - Żaklina. 
polonais $»^{24}$. Vu leur nombre et aussi la longueur de certaines d'entre elles (celle du GDF contient plus de 1000 unités et celle du GDP dépasse 1700 unités), un examen détaillé de leur contenu nécessiterait une étude à part et c'est pourquoi nous nous limitons ici à une description assez sommaire.

Tout d'abord, rappelons que dans deux dictionnaires anciens, les DA et JA, figure une seule liste de noms propres, réunissant des noms géographiques et des noms de personnes, parmi lesquels on retrouve des noms ethniques et des prénoms ( $c f$. point 4.5.). En ce qui concerne les informations véhiculées, la liste du JA constitue un cas limite : le nom français y est tout simplement suivi de son équivalent polonais (par ex. Paris, Paryż). Parmi les dictionnaires contemporains, il n'y a que le OX et le SM qui sont aussi minimalistes ( $c f$. Albania - Albanie, Afryka-Afrique (SM)).

Ensuite, le MB propose uniquement une liste des noms officiels des 22 régions françaises suivis de noms des villes qui s'y trouvent, pour la plupart leurs chefs-lieux. Tout comme c'est le cas pour les prénoms, ce dictionnaire ne donne aucun équivalent polonais de ces noms géographiques, si bien que la liste a un caractère purement encyclopédique. S'en démarque la liste du $\mathrm{OX}$ où les noms de 21 régions (la Corse étant absente), pas toujours cités dans leur forme complète (cf. Champagne ou Nord), sont quand même accompagnés des équivalents polonais (cf. Alsace - Alzacja), à moins que ces derniers n'existent pas (cf. FrancheComté $^{25}$ ). Dans trois dictionnaires PONS (POP, POS, POW), on présente non seulement la division administrative de la France métropolitaine, mais aussi celle de la Belgique et de la Suisse. Lorsqu'ils existent, les correspondants polonais des noms de régions, provinces ou cantons, et chefs-lieux administratifs sont donnés (par ex. la Flandre orientale - Flandria Wschodnia, Gand - Gandawa). Toujours est-il que ces listes ont un caractère ethnocentré : dans un bilingue comparant le français et le polonais, un renseignement sur la traduction des noms de divisions administratives polonaises serait tout aussi à sa place.

Dans les dictionnaires qui ne se limitent pas aux divisions administratives, les listes de noms géographiques recensent normalement plusieurs centaines d'unités, telles que des noms de pays, régions, continents, villes, mers et océans, fleuves et rivières, etc. Ces noms sont d'habitude suivis d'une abréviation précisant leur genre grammatical. En revanche, la transcription phonétique est rare. Elle n'est donnée systématiquement que dans le $\mathrm{CH}$ (pour les noms polonais) ainsi que dans les DU et le RO (pour les noms français) ${ }^{26}$.

De surcroît, neuf ouvrages incluent dans leurs listes des gentilés, donnés soit dans la liste même, au même titre que les toponymes, ce qui est le cas

${ }^{24}$ AS, p. 1059.

25 Le MB orthographie ce nom « Franche-Compte» (sic !).

${ }^{26}$ La transcription du RO devrait être entièrement revue, vu le nombre d'erreurs qu'elle contient. À titre d'exemple, la liste analysée informe que dans les noms tels que Cuba, Java, Jura ou Ouganda, le « a » final ne se prononce pas (cf. par ex. Cuba $[\mathrm{kyb}] f \mathrm{Kuba})$. 
des dictionnaires anciens (CA, CH, DA, JA ; par ex. Warszawa, warszawianin, Wenecja (CA)), soit à côté des toponymes respectifs (AS, DU, GDP, LARP, $\mathrm{SPO})^{27}$. Pourtant, cette solution, adoptée dans les dictionnaires contemporains, transforme petit à petit la liste en une succession d'articles, tout en faisant penser à une macrostructure à nids. Qui plus est, certains dictionnaires polonais-français ajoutent à la liste des adjectifs dérivés des noms propres, parce qu'en polonais, ces adjectifs n'ont pas la même forme que les gentilés respectifs ( $c f$. LARP : Litwa - Lituanie, litewski - lituanien, Litwin - Lituanien). Tout comme les gentilés, ils figurent directement dans la liste ( $c f$. DA) ou bien dans la microstructure de l'« article » construit autour du nom géographique ( $c f$. GDP, LARP).

Enfin, pour les dictionnaires en deux parties, polonais-français et français-polonais, se pose la question de savoir s'il suffit de donner une liste des noms géographiques, allant par exemple du français vers le polonais, ou bien s'il en faut deux. À ce propos, seuls les RO et SPO se limitent à une liste, partant des noms français, tandis que les BU, CA, DU, JST et SZT en proposent deux, ce qui est sans doute une meilleure solution.

\section{REMARQUES FINALES}

Les listes de noms propres constituent un élément facultatif, mais relativement fréquent de la mégastructure des dictionnaires généraux polonais-français et français-polonais : il y en a au moins une dans la moitié des ouvrages consultés. Bien entendu, l'absence d'une telle liste ne signifie pas du tout que les noms propres soient bannis du dictionnaire, car ils font alors partie de sa nomenclature. D'ailleurs, les dictionnaires qui ont recours aux listes départagent le plus souvent les noms propres en ceux listés et ceux donnés dans la nomenclature. C'est ainsi que le DPP ou le RO placent les noms de pays en annexe tandis que les noms de leurs habitants sont à chercher dans le corps du dictionnaire. En outre, il arrive parfois que les deux ensembles de noms, celui de la liste et celui de la nomenclature, se recoupent partiellement, ce qui laisse l'impression d'un manque de cohérence.

Ensuite, il n'existe pas toujours de correspondance entre une classe référentielle de noms propres et une liste donnée. En effet, si certains phénonymes, ou les signes du zodiaque, font l'objet d'une liste à part, nous n'avons relevé aucune liste réservée exclusivement aux noms ethniques, car ces anthroponymes sont régulièrement cités dans les listes de toponymes.

Quant à la valeur informative des listes de noms propres, nous avons vu qu'en plus de renseigner sur la correspondance orthographique, elles peuvent inclure la prononciation, le genre et le nombre, une caractéristique stylistique et même un ajout encyclopédique. Toutefois, l'accumulation de toutes ces informations utiles,

${ }^{27}$ Aucun dictionnaire du corpus ne répertorie les noms d'habitants dans une liste à part. 
pour ne pas dire indispensables, aux usagers d'un bilingue risque de transformer la liste en une succession de vrais articles de dictionnaire. D'où la remarque répétée tout au long de cet article : rien n'empêche d'introduire les noms propres dans le corps du dictionnaire et de les traiter à l'égal des noms communs, en n'oubliant pas l'aspect syntaxique, forcément négligé dans les listes du fait qu'il n'y a pas de place pour des exemples d'emploi.

Autrement, il faut souligner que pour son usager, un dictionnaire est une autorité, si bien qu'il devrait fournir une description exhaustive et fiable. Or, dans l'immense majorité des cas, les lexicographes négligent l'aspect phonétique des noms propres, comme si leurs dictionnaires ne servaient qu'à la compréhension ou production écrites. En plus, dans certains ouvrages soumis à l'analyse, outre des cas discutables dus aux contours flous de la notion de nom propre et, partant, à un usage écrit parfois flottant, nous avons relevé un certain nombre d'erreurs orthographiques ou de transcription phonétique, sans parler des équivalences problématiques ou tout simplement absentes. Plutôt que de multiplier des listes, consacrées par exemple aux noms d'alcools, au zodiaque ou aux planètes du système solaire, il serait donc préférable de veiller à leur qualité, sans laquelle un bilingue ne pourra pas remplir sa fonction de façon satisfaisante. Sur ce point, nos considérations convergent d'ailleurs avec celles de Mariadomenica Lo Nostro qui, au terme de son analyse des dictionnaires français-italien et italien-français, constate « qu'il n'est pas rare que, feuilletant les paratextes, on perçoive encore la sensation que les choix tombent sur un besoin de nouveauté, plutôt que sur un réel but didactique, capable d'accomplir la tâche de médiation lexicale $»^{28}$.

\section{DICTIONNAIRES DU CORPUS}

AS : Dictionnaire Assimil Kernerman polonais-français, français-polonais, Assimil, Chennevièressur-Marne 2009.

BER : M. Zając, Dictionnaire français-polonais, polonais-français Berlitz, Langenscheidt, Warszawa [s.d.].

BI : L. Bielas, Dictionnaire minimum français-polonais, polonais-français, Wiedza Powszechna, Warszawa [1964] 1987 (7éd.).

BU : Stownik francusko-polski, polsko-francuski, Buchmann, Warszawa 2008.

CA : O. Callier, Dictionnaire de poche français-polonais et polonais-français, Otto Holtze's Nachfolger, Leipzig [1906] 1923 (5éd.).

CH : A.M. Chmurski, Dictionnaire Polonais-Français, Librairie Garnier Frères, Paris 1940.

DA : P. Dahlmann, Nouveau dictionnaire de poche polonais-français et français-polonais, $1^{\text {ière }}$ partie : polonais-français, B. Behr's Verlag, Berlin ca 1913 (10 éd.).

DEF : J. Sikora-Penazzi, K. Sieroszewska, Dictionnaire élémentaire français-polonais, Wiedza Powszechna, Warszawa 1997.

DEP : J. Sikora-Penazzi, K. Sieroszewska, Dictionnaire élémentaire polonais-français, Wiedza Powszechna, Warszawa 1997.

${ }^{28}$ M. Lo Nostro, op. cit., p. 216. 
DPF : K. Kupisz, B. Kielski, Dictionnaire pratique français-polonais avec supplément, Wiedza Powszechna, Warszawa [1968] 1982 (4e éd.).

DPP : K. Kupisz, B. Kielski, Dictionnaire pratique polonais-français avec supplément, Wiedza Powszechna, Warszawa [1969] 1993 (9e éd.).

DU : M. Słobodska, Dictionnaire universel français-polonais et polonais-français, Delta W-Z, Warszawa 2001.

GDF : J. Dobrzyński et al., Grand dictionnaire français-polonais, Wiedza Powszechna, Warszawa [1980-1982] 1991 (4éd.).

GDP : Grand dictionnaire polonais-français, t. I-V, Wiedza Powszechna, Varsovie 1995-2008.

HAF : B. Hamel, Dictionnaire de poche français-polonais et polonais-français avec prononciation phonétique, $1^{\text {ère }}$ partie : français-polonais, Trzaska, Evert \& Michalski, Librairie Polonaise à Paris [s.d.].

HAP : B. Hamel, Dictionnaire de poche français-polonais et polonais-français avec prononciation phonétique, $2^{\mathrm{e}}$ partie : polonais-français, Trzaska, Evert \& Michalski, Librairie Polonaise à Paris [s.d.].

JA : W. Janusz, Dictionnaire complet français-polonais et polonais-français, partie française, E. Winiarz, Léopol 1891 ( $3^{\mathrm{e}}$ éd.).

JST : A. Jedlińska, L. Szwykowski, J. Tomalak, Dictionnaire de poche français-polonais, polonais-français, Wiedza Powszechna, Varsovie [1979] 1984 (3 éd.).

KA : P. Kalina, Dictionnaire français-polonais et polonais-français, Czytelnik, Warszawa 1949.

KS : W. Kwiatkowski, K. Sobczyński, Dictionnaire français-polonais, polonais-français, ABC Future, Kraków 1997.

KU : A. Kuźnik, Dictionnaire français-polonais, polonais-français, Wydawnictwo Językowe Aneks, Wałbrzych 2001.

LAN : Duży słownik polsko-francuski, francusko-polski, Langenscheidt, Warszawa 2012.

LAP : B. Assaf (dir.), Dictionnaire de poche français-polonais, polonais-français, Larousse, Paris 2005.

LARF : Dictionnaire Compact plus français-polonais, Larousse/Rea, Warszawa 2001.

LARP : Dictionnaire Compact plus polonais-français, Larousse/Rea, Warszawa 2003.

LAW : B. Assaf (dir.), Stownik polsko-francuski, francusko-polski, Larousse, Wrocław 2006.

LIF : A. Lipska, Szkolny stownik francusko-polski, WSiP, Warszawa 1992.

LIP : A. Lipska, Szkolny stownik polsko-francuski, WSiP, Warszawa 1992.

MB : B. Meister, D. Botton, Dictionnaire polonais-français, français-polonais, Ex Libris, Varsovie 2002.

MC : Z. Matkowski, S. Ciesielska-Borkowska, Dictionnaire encyclopédique français-polonais et polonais-français, $1^{\text {ère }}$ partie : français-polonais, Librairie Bernard Połoniecki, LwowVarsovie 1928.

NM : A. Nowak, M. Musiał, Uniwersalny stownik francusko-polski i polsko-francuski, Liberal, Kraków 2000.

OX : V. Grundy, J. Barnes, K. Podracka, Stownik francusko-polski, polsko-francuski, Delta W-Z, Warszawa 2002.

POM : A. Stanisławska et al., Słownik mini francusko-polski, polsko-francuski Pons, LektorKlett, Poznań 2004.

POP : B. Chełkowska et al., Praktyczny słownik francusko-polski, polsko-francuski Pons, LektorKlett, Poznań 2011.

POS : B. Chełkowska et al., Szkolny stownik francusko-polski, polsko-francuski Pons, LektorKlett, Poznań 2006.

POU : A. Stanisławska, Uniwersalny słownik francusko-polski, polsko-francuski Pons, LektorKlett, Poznań 2004. 
POW : B. Chełkowska et al., Współczesny stownik francusko-polski, polsko-francuski Pons, LektorKlett, Poznań 2007.

PWN : G. Migdalska, Stownik francusko-polski, polsko-francuski, Wydawnictwo Naukowe PWN, Warszawa 2003.

RO : M. Romanowska, Kieszonkowy stownik francusko-polski, polsko-francuski, Wydawnictwo Zielona Sowa, Kraków 2007.

SM : M. Słobodska, Mini dictionnaire français-polonais, polonais-français, Harald G, Warszawa 2000.

SPO : M. Słobodska, Język francuski na co dzień. Francusko-polski, polsko-francuski słownik popularny, Delta W-Z, Warszawa 1997.

SPR : Sprytny stownik francusko-polski i polsko-francuski, Lingea, Kraków 2010.

SS : Szkolny słownik francusko-polski, polsko-francuski, Faktor, Poznań 2005.

SZT : L. Szwykowski, J. Tomalak, Petit dictionnaire français-polonais, polonais-français, Wiedza Powszechna, Varsovie 1970.

\title{
LISTS OF PROPER NOUNS IN POLISH-FRENCH AND FRENCH-POLISH GENERAL DICTIONARIES
}

\author{
Summary
}

The paper deals with the lists of proper names found in 22 Polish-French and/or French-Polish dictionaries out of the 45 dictionaries investigated, including dictionaries of different sizes published over the last 150 years. The lists most often include geographical names, gentilics and first names. A popular practice is to include certain items in the main body of the dictionary and to list other items in appendices; sometimes, these two sets of proper names overlap, which is incoherent. In addition to the quite evident information on spelling, the analysed lists may provide phonetic, grammatical, stylistic or encyclopedic data. However, the accumulation of such information, very valuable for users, transforms the lists into a succession of entries. That is why, instead of striving for originality by putting together lists of such exotic terms as Zodiac signs or celestial bodies, it would be better to incorporate proper names into the macrostructure.

Key words: Polish-French dictionaries, French-Polish dictionaries, megastructure, list of proper names. 